\title{
Primary testicular carcinoid tumor with marked lymphovascular invasion
}

\author{
Hyun Jung Lee ${ }^{1,2}$, Joon Young Park ${ }^{1}$, So Young Kim ${ }^{1}$, Chung Su Hwang ${ }^{1}$, Jung Hee Lee ${ }^{1}$, Dong Hoon Shin ${ }^{1}$, Jee Yeon Kim ${ }^{1,2}$ \\ ${ }^{1}$ Department of Pathology, School of Medicine, Pusan National University, Yangsan; \\ ${ }^{2}$ The Research Institute for Convergence of Biomedical Science and Technology, Pusan National University Yangsan Hospital, Yangsan, Korea
}

\begin{abstract}
Testicular carcinoid tumors are very rare, accounting for less than $1 \%$ of all testicular tumors. We report a rare case of a testicular carcinoid tumor with extensive lymphatic invasion. A 42-year-old man presented with a painless, enlarged right testicular mass. There was no history of injury or discomfort in this region. Right radical orchiectomy was performed, which showed a well-defined, non-encapsulated solid white mass with calcification $(7.0 \times 4.5 \times 3.5 \mathrm{~cm})$ and absence of cystic components. Microscopic examination using hematoxylin and eosin staining of the tumor sections identified organoid, trabecular, and solid patterns with rosette formation. Extensive multifocal lymphatic invasion was observed. Immunohistochemistry was positive for synaptophysin, chromogranin, and CD56. Testicular carcinoid tumors usually show good prognoses; however, there was extensive lymphovascular invasion in this case. Thus, in the case of unusual presentation of the disease, close follow-up is necessary.
\end{abstract}

Key Words: Testicular carcinoid tumor; Testicular neoplasm; Lymphovascular invasion; Orchiectomy

Received: August 18, 2021 Revised: September 7, 2021 Accepted: September 10, 2021

Corresponding Author: Jee Yeon Kim, MD, Department of Pathology, Pusan National University School of Medicine, 49 Busandaehak-ro, Mulgeum-eup, Yangsan 50612, Korea Tel: +82-55-360-1861, Fax: +82-55-360-1865, E-mail: jeykim@pusan.ac.kr

Testicular carcinoid tumors are very rare, with a prevalence of less than $1 \%$ among all testicular tumors [1]. The first case of testicular carcinoid tumor was reported as an element of a benign cystic teratoma by Simon et al. in 1954 [2]. Approximately $10 \%$ of testicular carcinoid tumors are complicated by the carcinoid syndrome $[3,4]$. Testicular carcinoid tumors can be divided into three subgroups: primary testicular carcinoid tumors, carcinoid tumors associated with teratoma, and carcinoid metastasis to the testis [5].

Carcinoid tumors were initially considered benign [6]; however, they are now regarded as potentially malignant neoplasms [7]. Furthermore, benign and malignant carcinoid tumors are difficult to differentiate histologically, making it important to identify metastases. The preferred therapy for carcinoid tumors is surgical excision. Other therapies include somatostatin ana$\operatorname{logs}$, interferon- $\alpha$, chemotherapeutics, and radiation. The treatment with somatostatin analogs is limited to symptom control in patients with carcinoid syndrome and stabilization of disease progression.

There are only a few studies on testicular carcinoid tumors, and the clinical implications of its diagnosis remain unclear. We report a very rare case of a carcinoid tumor of the testis with extensive lymphatic invasion but no manifestation of carcinoid syndrome.

\section{CASE REPORT}

A 42-year-old man presented with a painless, enlarged right testicular mass confirmed by ultrasonography $(7.0 \times 4.5 \times 3.5 \mathrm{~cm}$ in size). The patient did not have a history of injury or discomfort in this region. A computed tomography scan revealed a $7.0 \mathrm{~cm}$ homogenous enhancing mass with calcification in the right testicle. Results of laboratory analysis were as follows: $\alpha$-fetoprotein (AFP), $5.03 \mathrm{ng} / \mathrm{mL}$ (normal range, 0.1 to $930 \mathrm{ng} / \mathrm{mL}$ ), beta-human chorionic gonadotropin, $<0.5 \mathrm{mIU} / \mathrm{mL}$ (normal range, 0 to $5 \mathrm{mIU} / \mathrm{mL}$ ); and testosterone, $1.83 \mathrm{ng} / \mathrm{mL}$ (normal range, 1.2 to $10.19 \mathrm{ng} / \mathrm{mL})$.

Right radical orchiectomy was performed, and it revealed a well-defined, non-encapsulated solid white mass with calcification $(7.0 \times 4.5 \times 3.5 \mathrm{~cm})$ and no cystic components. The cut surface was clear and evenly white, with no hemorrhage or necrosis (Fig. 1). The spermatic cord did not show any abnormalities. Following the surgery, the patient has been alive and well for 13 

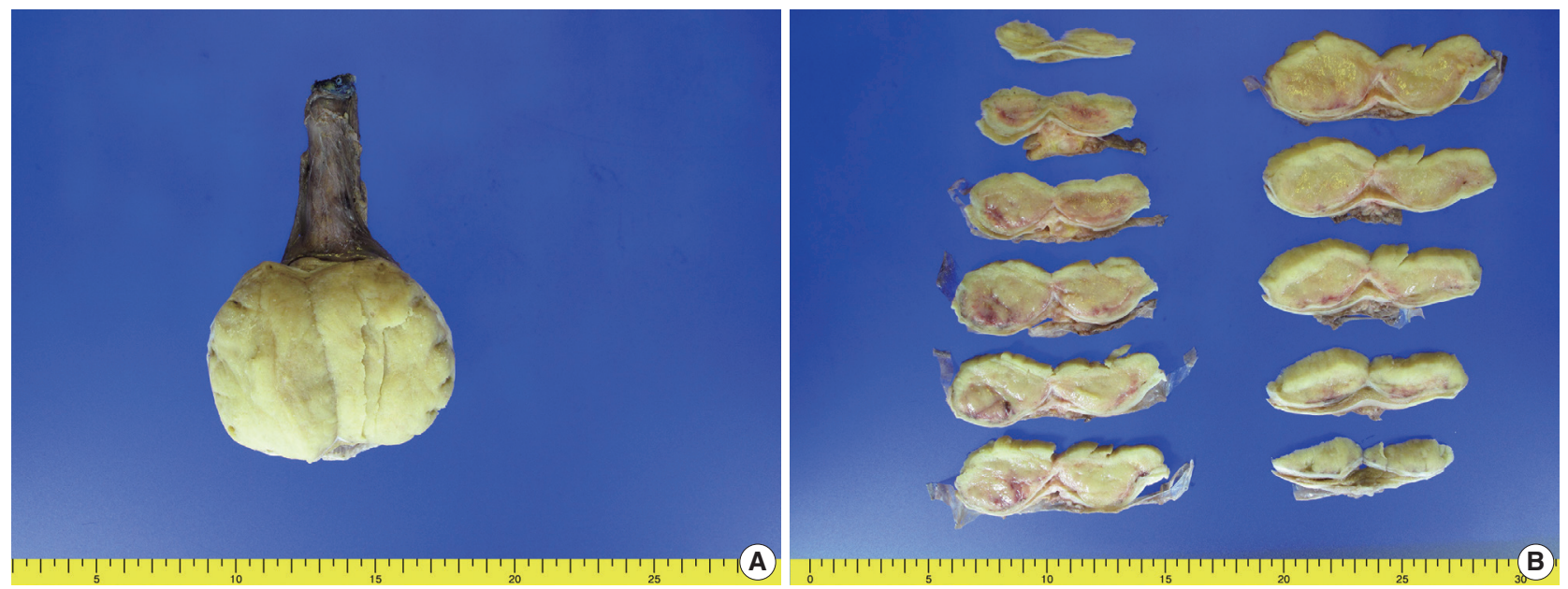

Fig. 1. Gross appearance of the right testicular mass. (A) A well-defined, non-encapsulated solid white mass is observed. (B) The cut surface is clear and evenly white without hemorrhage or necrosis.
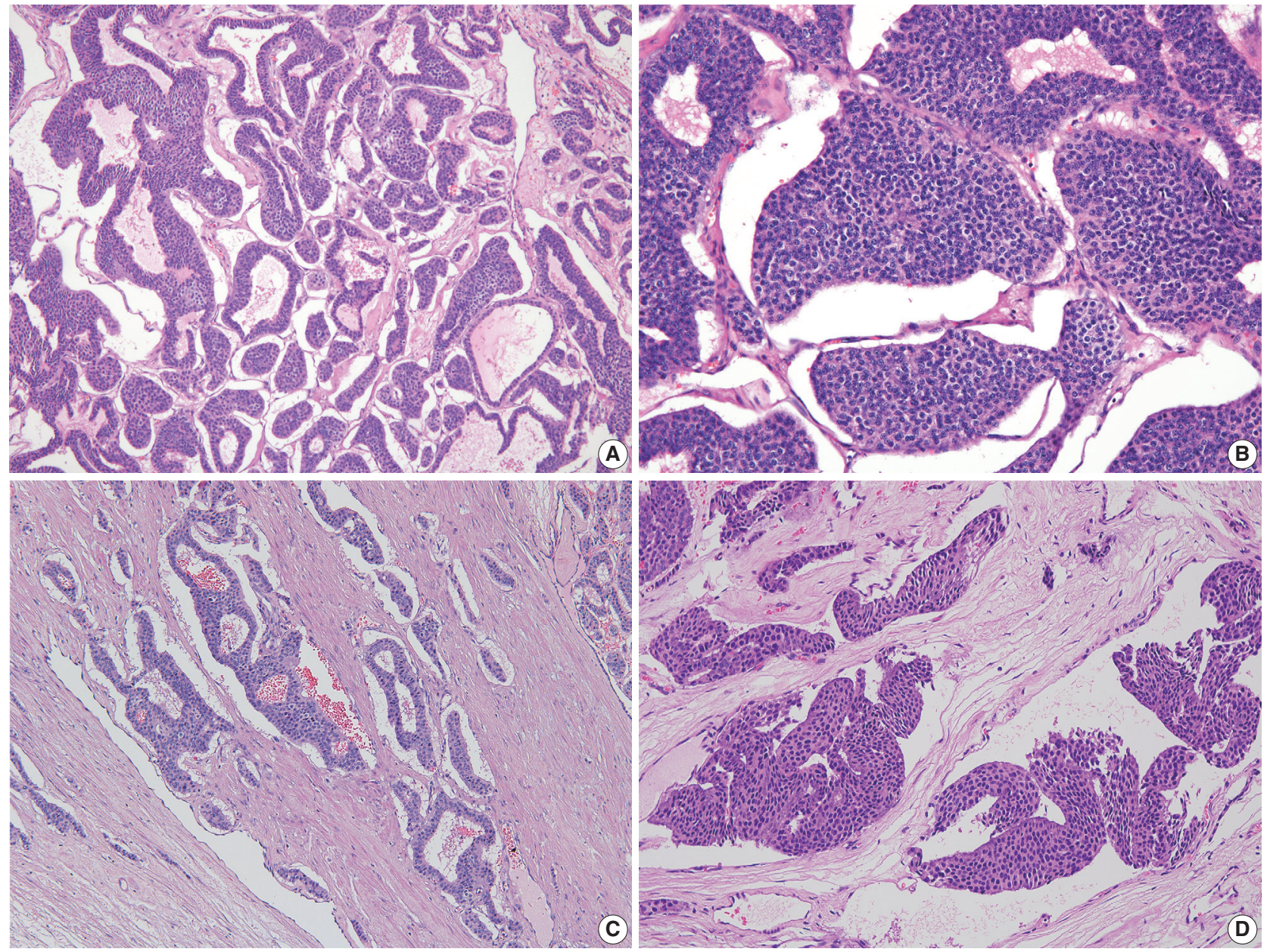

Fig. 2. Microscopic findings of the testicular carcinoid tumor. (A) Organoid, trabecular, and solid patterns are visible. (B) The cells have a uniform polygonal appearance with fine granular cytoplasm and round nuclei containing fine chromatin and small nucleoli. (C, D) Extensive multifocal lymphatic invasion is detected. 
months without lymph node metastasis or recurrence.

On microscopy, a hematoxylin and eosin stained tumor section showed organoid, trabecular, and solid patterns with rosette formation. The cells had a uniform polygonal shape with fine granular cytoplasm and round nuclei containing fine chromatin and small nucleoli (Fig. 2A, B). The mitotic figure was 0 per highpower field (HPF). Ki67 proliferation index was less than 1\%, and tumor necrosis was absent. Extensive multifocal lymphovascular invasion was identified (Fig. 2C, D). There were no germ cell neoplasia in situ or other germ cell tumor components and no teratomatous component. The tumor had invaded the rete testis (pT2). Hemorrhage and necrosis were not observed. Further evaluation by immunohistochemistry showed the tumor was positive for synaptophysin, chromogranin, and CD56 (Fig. 3A, B) and negative for GATA3, OCT3/4, CD30, AFP, and c-kit. CD31 showed positivity in the lymphovascular endothelium (Fig. 3C), and D2-40 showed positivity in the lymphatic endothelium (Fig.
3D). Based on the morphological and immunohistochemical findings, this case was diagnosed as primary testicular carcinoid tumor. The tumor was staged as pT2N0M0.

\section{DISCUSSION}

The incidence of testicular tumors is low, and testicular carcinoid tumors account for only $1 \%$ of all testicular tumors [8]. Primary testicular carcinoid tumors are exceedingly rare, and only a few cases have been reported in the literature. These tumors present in patients typically between their second and ninth decades of life, with an average diagnosis age of 46 years $[9,10]$. Testicular carcinoid tumors often do not elicit symptoms, or they cause symptoms similar to those of other testicular tumors. The symptoms are not specific, and the carcinoid syndromes are often not recognized until the histological diagnosis is confirmed.

In contrast to most ovarian carcinoid tumors that present as
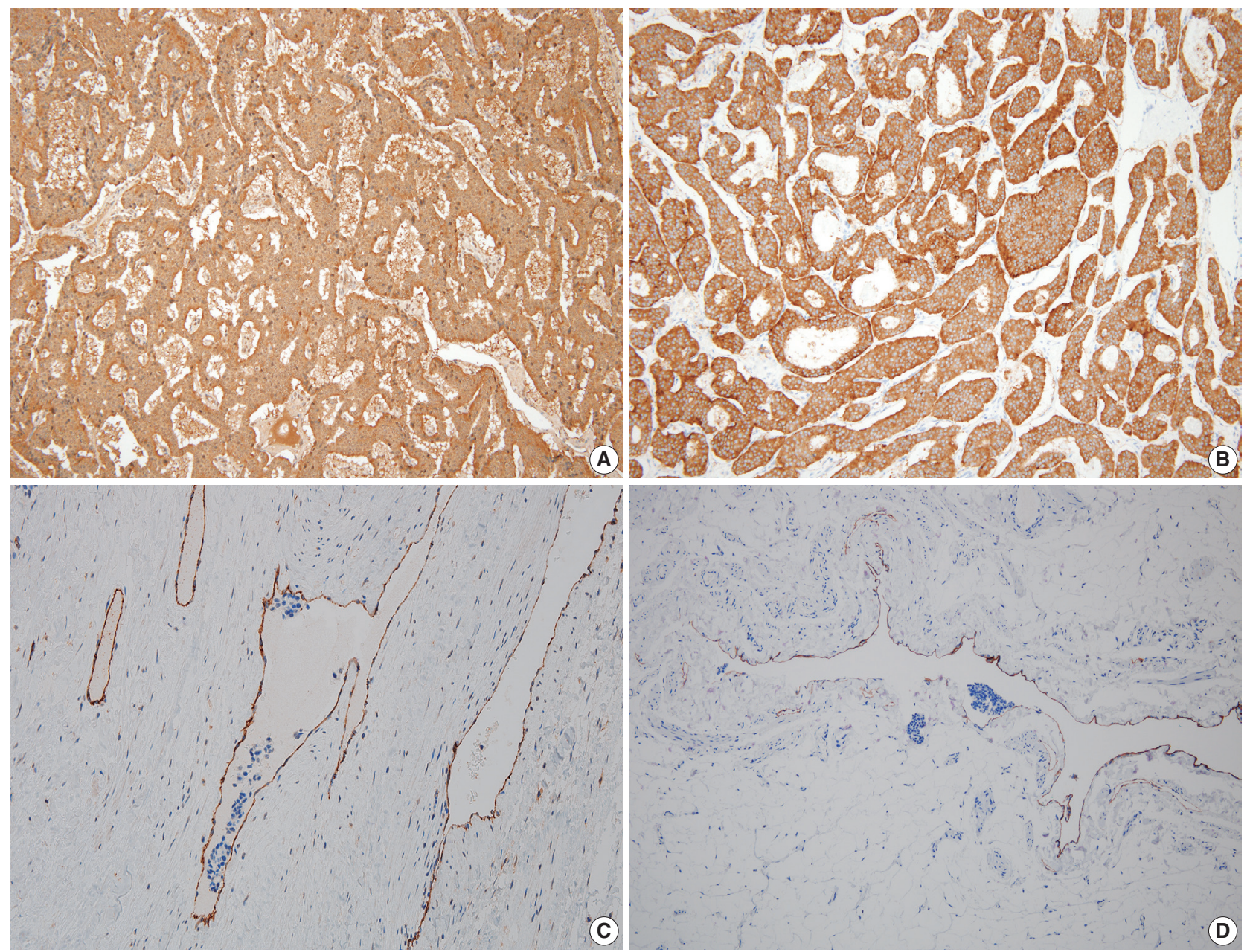

Fig. 3. Immunohistochemical staining is positive for chromogranin and (A) synaptophysin (B). (C) CD31 shows positivity for lymphovascular endothelium. (D) D2-40 shows positivity for lymphatic endothelium. 
teratomatous components, most testicular carcinoid tumors are histologically pure [11,12]. Carcinoid tumors are 15 times more common in the ovaries than in the testicles. Ovarian carcinoid tumors are most often associated with mature cystic teratomas, which constitute the most common type of ovarian germ cell tumors in females up to 30 years of age [13].

Histogenesis of testicular carcinoid tumors has not yet been defined. There is a strong rationale that testicular carcinoid tumors are of germ cell origin. Most tumor cases in the testes, especially solitary, large tumors, are likely to be primary. Testicular tumors are considered primary when metastasis from another primary tumor has been reasonably ruled out. Malignant outcomes have been reported in approximately $16 \%$ of cases. Features correlated with adverse outcomes include coagulative necrosis, 2 to 10 mitoses per $10 \mathrm{HPF}$, atypical mitoses, and atypical carcinoid tumor-like features [14]. The present case was primary testicular carcinoid tumor, and no metastasis was identified. Coagulative necrosis and mitosis, which are usually seen in atypical carcinoid tumor, were not identified. Compared to previously reported cases, this tumor showed extensive lymphovascular invasion without atypical carcinoid tumor pattern.

The key features of testicular carcinoid tumors are organoid and trabecular patterns, with tumor cells arranged in solid nests or cords. The tumor cells have uniform polygonal shapes with fine granular cytoplasm and round nuclei containing fine chromatin and small nucleoli.

Testicular carcinoid tumors usually have a good prognosis; however, when there is an unusual presentation of the disease, such as extensive lymphatic invasion as in this case, a close follow-up is necessitated. The presence of multifocal lymphovascular invasion in this case was striking and warranted close clinical follow-up and further work-up.

This is the first report of a primary testicular neuroendocrine tumor with extensive lymphatic invasion. Additionally, patients with malignant tumors exhibit a prolonged clinical course before death, which usually results from distant metastases. Therefore, a long-term follow-up evaluation is necessary for patients with this neoplasm.

\section{Ethics Statement}

This study was approved by the Institutional Review Board of the Pusan National University Yangsan Hospital (05-2021-173), and the need for informed consent was waived.

\section{Availability of Data and Material}

All data generated or analyzed during the study are included in this published article.

\section{Code Availability \\ Not applicable.}

ORCID

Hyung Jung Lee https://orcid.org/0000-0002-2995-6060 Joon Young Park https://orcid.org/0000-0002-4193-2520 So Young Kim https://orcid.org/0000-0003-3076-9147

Chung Su Hwang https://orcid.org/0000-0001-9520-0301 Jung Hee Lee https://orcid.org/0000-0003-3003-2217

Dong Hoon Shin https://orcid.org/0000-0002-4980-9295

Jee Yeon Kim https://orcid.org/0000-0002-0503-984X

\section{Author Contributions}

Conceptualization: HJL. Data curation: JYP, HJL. Formal analysis: SYK, HJL. Investigation: CSH, HJL. Validation: JHL, HJL. Visualization: HJL. Writing_original draft: HJL. Writing_review \& editing: HJL, DHS, JYK. Approval of final manuscript: all authors.

\section{Conflicts of Interest}

The authors declare that they have no potential conflicts of interest.

\section{Funding Statement}

This study was supported by a 2020 research grant from Pusan National University Yangsan Hospital.

\section{References}

1. Ulbright TM, Young RH. Carcinoid tumor of the testis. Am J Clin Pathol 2004; 121: 297.

2. Simon HB, Mc DJ, Culp OS. Argentaffin tumor (carcinoid) occurring in a benign cystic teratoma of the testicle. J Urol 1954; 72: 892-4.

3. Singer AJ, Anders KH. Primary carcinoid of the testis 25 years after contralateral testicular seminoma. Urology 2001; 57: 554-5.

4. Zavala-Pompa A, Ro JY, el-Naggar A, et al. Primary carcinoid tumor of testis. Immunohistochemical, ultrastructural, and DNA flow cytometric study of three cases with a review of the literature. Cancer 1993; 72: 1726-32.

5. Stroosma OB, Delaere KP. Carcinoid tumours of the testis. BJU Int 2008; 101: 1101-5.

6. Lu C, Zhang Z, Jiang Y, et al. Primary pure carcinoid tumors of the testis: Clinicopathological and immunophenotypical characteristics of 11 cases. Oncol Lett 2015; 9: 2017-22.

7. Soga J. Carcinoids and their variant endocrinomas: an analysis of 11842 reported cases. J Exp Clin Cancer Res 2003; 22: 517-30.

8. Sun GH, Fang DB, Shen ZJ, Cai SL. Diagnosis of the testicular tumor (57 case reports). Zhonghua Nan Ke Xue 2003; 9: 364-6.

9. Kato N, Motoyama T, Kameda N, et al. Primary carcinoid tumor of the testis: immunohistochemical, ultrastructural and FISH analysis with review of the literature. Pathol Int 2003; 53: 680-5.

10. Reyes A, Moran CA, Suster S, Michal M, Dominguez H. Neuroendocrine carcinomas (carcinoid tumor) of the testis: a clinicopathologic and immunohistochemical study of ten cases. Am J Clin Pathol 2003; 120: $182-7$.

11. Frank RG, Gerard PS, Anselmo MT, Bennett L, Preminger BI, Wise GJ. Primary carcinoid tumor of the testis. Urol Radiol 1991; 12: 203-5.

12. Talerman A, Gratama S, Miranda S, Okagaki T. Primary carcinoid tumor of the testis: case report, ultrastructure and review of the literature. Cancer 1978; 42: 2696-706. 
13. Roth LM, Talerman A. Recent advances in the pathology and classification of ovarian germ cell tumors. Int J Gynecol Pathol 2006; 25: 305-20.
14. Wang WP, Guo C, Berney DM, et al. Primary carcinoid tumors of the testis: a clinicopathologic study of 29 cases. Am J Surg Pathol 2010; 34: 519-24. 